\title{
Anthropomorphic robotic hand for teleoperation \& telepresence in space \& hazardous environments
}

\author{
Mihail Teodorescu ${ }^{1}$, Lucian $_{\text {Milea }}{ }^{2}$, Monica Dascalu $^{3}$, Dan Coroama $^{3}$ \\ ${ }^{1}$ Research Institute for Artificial Intelligence, Bucharest, Romania \\ ${ }^{2}$ Solaris Consult S.R.L., Bucharest, Romania \\ ${ }^{3}$ Politehnica University of Bucharest, Romania
}

Email address:

mihait@artsoc.ro (M. Teodorescu), lucian@artsoc.ro (L. Milea), monicad@artsoc.ro (M. Dascalu), dcoroama@artsoc.ro (D. Coroama)

To cite this article:

Mihail Teodorescu, Lucian Milea, Monica Dascalu, Dan Coroama. Anthropomorphic Robotic Hand for Teleoperation \& Telepresence in Space \& Hazardous Environments. International Journal of Astrophysics and Space Science. Vol. 1, No. 5, 2013, pp. 64-69.

doi: 10.11648/j.ijass.20130105.11

\begin{abstract}
The research presented in this article aimed at achieving an innovative anthropomorphic robotic arm with intelligent sensory feedback, which is remotely controlled by a human operator, using a control-glove. The movements of the human operators hand will be exactly reproduced by the manipulation robotic hand, which also provides feedback regarding the pressure confronted with and 3D visual information of the manipulation area. This will enable the possibility of complex and high precision manipulation in unfriendly and hazardous environments, without exposing the human operator to risks. Teleoperation will be carried out by means of a Multi-sensory control glove (MSG), which will enable high-precision tasks performing with almost no preliminary training required for the operator (the robotic hand having all five articulated and driven fingers and allowing human-like complex maneuvers). The MSG is equipped with position movement and acceleration sensors, and also a system for transmitting the haptic feedback to the operator, which is the first component of the telepresence implemented in the robotic system. The second component is 3D visual feedback from the operation site, using a stereoscopic HD camera mounted on the robot platform and a 3D vision helmet, with glasses containing two OLED displays for the operator. Using this kind of robotic system, the human operator can act efficiently from inside safe environment, only the robotic hand and it's mobile platform (or supporting arm) being exposed to hazardous conditions. The applications of this robotic hand could include the following domains: space exploration and working (remote operations on void space or on other planets' surface driven from human-safe environment), working in toxic atmosphere (chemically poisoned or toxic gas emanation sites); working in high radiation-level environments (like nuclear plants); working in marine applications (deep ocean exploration, repair of offshore oil platforms etc.) etc.
\end{abstract}

Keywords: Robotic Hand, Space Teleoperation, Telepresence, 3D Vision, Haptic Feedback, Hazardous Environments, Multisensory Feedback

\section{Introduction}

All over the world, in recent years, intelligent robotic arms and hands have enjoyed intense focus of attention, which is visible in the ever increasing number of international research projects and scientific articles in specialized journals worldwide [1], [2]. Systematic efforts made by many inter-disciplinary specialist research teams lead to significant annual progress that enriches the market with better performing robotic arms and hands [3], [4].

A robotic arm is a type of mechanical arm, programmable or remotely controlled, with similar functions to a human arm [5]; the arm may be the whole system or may be part of a more complex robot [6], [7]. The links of such a manipulator are connected by joints allowing either rotational motion (such as in an articulated robot) or translational (linear) displacement. The links of the manipulator can be considered to form a kinematic chain [8]. The terminus of the kinematic chain of the manipulator is called the end effector and it is analogous to the human hand [9]. In various circumstances, for the end effector a close emulation of the human hand becomes necessary [10], [11].

A robotic hand, can replace a human that should execute certain operations in hazardous environments and/or hard-to-reach places, wherever a live-threatening or risky situation appears [12], [13]. Some examples of applications 
could be the following:

Working in toxic atmosphere - chemically poisoned or toxic gas emanation sites [14]. In this case, the robotic system carrying the robotic hand can be also equipped with specialized sensors transmitting the type and concentration of dangerous substances present [15].

Working in high radiation-level environments, like nuclear plants [16]. Remote manipulators are used to handle radioactive materials or to execute repairs or other necessary operations in environments with dangerous radiation levels.

Marine applications. Marine remotely operated vehicles (ROVs) are widely used to work in water too deep or too dangerous for divers. They repair offshore oil platforms and attach cables to sunken ships to hoist them. They are usually attached by a tether to a control center on a surface ship [17].

Working in space - this is the most interesting use of remote controlled robotic arms comprising teleoperation \& telepresence [18], [19]. Several robotic arms suitable for space applications were designed and developed, with focus on size, force capabilities and dexterity. The European Robotic Arm ERA was designed for the International Space Station (ISS) in order to transfer small payloads directly from inside to outside the Station [20]. Another task for ERA is to transport astronauts to a position where they can work on the exterior of the Space Station, or from one external location to another. This saves valuable time and effort during spacewalks [21].

The joints of such robotic arms are complex mechatronic devices that integrate all the mechanical, electronics and computing elements needed to achieve high precision/high torque rotary motion, but only selected tasks with specialized tools can be carried out by the robotic arm. More complex, unforeseen tasks can be carried out only by astronauts, but allways imply a certain amount of risk [22].

Also for planetary explorations, robot arms mounted on a vehicle were designed and, in the case of ESA's Eurobot Ground Prototype (EGP), the system can work on a planet's surface either autonomously or in cooperation with astronauts. It has a pair of robotic arms with interchangeable tools, force and torque sensors and advanced vision systems, including a 3D camera [23]. It can also transport an astronaut, who can operate it by simple voice commands or a joystick. An anthropomorphic end effector is also in this case missing.

An example of force-feedback manipulation designed for space applications is the ESA Human Arm Exoskeleton, a wearable human-machine-interface that allows to operate a remote robot arm through haptic telepresence. With haptic telepresence a robot can be operated efficiently even without training.

The exoskeleton transmits commands and receives feedback regarding the position and motion of the shoulder, elbow and wrist joints.

Although such applications were designed by NASA and ESA and are expected to be implemented at the International Space Station (ISS), up to now they are not fully functional in space.
The main problem of the currently used robotic hands consists in movement limitations and in constraints regarding the control possibilities. Another main limitation is the lack of sensory feedback that would be desired for control actions and adjustment of the dynamic force exerted. The accuracy of the motions performed by a human operator is limited by the protective suite he is wearing and thus the operations he can perform are restricted.

\section{Proposed Solution \& Methodology}

In this article we consider the actions carried out by existing robotic arms of these joints to be primary positioning actions, and we focus on the end-effector conceived as a anthropomorphic hand with controllable wrist and the finger joints. A robotic hand with human-like degrees of freedom will dramatically extend the possibilities of intervention and handling, without exposing humans to any risk.

\subsection{The Proposed Solution}

The authors aimed at achieving an innovative robotic hand with intelligent sensory feedback, which is remotely controlled by a human operator, using a control-glove. The movements of the operators arm and hand will be exactly reproduced by the robotic system, which provides feedback regarding the pressure confronted with and also 3D visual information of the manipulation area. This will enable the possibility of complex and high precision manipulation in unfriendly and hazardous environments, without exposing the human operator to risks. The use of a control-glove will assure performing high-precision tasks with almost no preliminary training required for the operator (the robotic hand having all five articulated and driven fingers and allowing human-like complex maneuvers). The Multi-sensory control glove (MSG) is equipped with position and movement sensors, and also a system for transmitting the pressure-feedback to the operator. Stereoscopic vision of the manipulation site will be perceived by the operator wearing specialized $3 \mathrm{D}$ viewer with two active displays. Using this robotic system, the human operator can act from inside safe environment, only the robotic hand being exposed to hazardous conditions.

\subsection{Teleoperation \& Telepresence Functions}

Teleoperation, indicating operation of a machine at a distance, implies the existence of a human-machine interface, a transmission system, a control system, and a executing robotic system [12], [13].

On the other hand, telepresence is the capability of the robotic system to feed back realistically sensory data from a remote site or even a virtual world. Haptic feedback and stereo vision feedback are brought together in this article, offering effective sensory data, in real time, to the remote operator [23]. 


\subsection{Implementation Methodology}

The interface proposed in this article is based on a control glove that senses the movements of the human operator, but also provides tactile feedback from the robotic hand (Multisensory Control Glove - MSG). The MSG transduces every movement of the operators hand into specific electrical signals, which are transmitted to the robotic hand, which duplicates these movements. A microprocessor-based control system supervises the transmission of the movement signals to the robotic hand and also the feedback signals. An advantage of using a multisensory control-glove lies in the fact that it is equipped with position sensors and accelerometers, which makes it possible for each movement to be translated into precise motion algorithms, regardless of its complexity. This original modality of obtaining the motion algorithms for all the mobile elements of the artificial hand is highly effective and does no longer require the complex and difficult stages necessary for describing movement equations.

Due to the intelligent control system, certain repetitive movements or operations can be pre-programmed and triggered as desired. Also, other unwanted movements, even if accidentally performed by the human operator, can be blocked or may require an additional validation from the operator, if so programmed. This feature can help avoiding application-specific accidents caused by human errors, significantly improving the quality and safety of the operations carried out.

Experiments performed with the MSG are also being put to good use in identifying the optimum number of freedom degrees of the mechanical structure, the number of motor elements, their layout and the power needed for performing each succession of movements by the robotic hand.

The control function is distributed into sub-systems of lower complexity for each component, simultaneously creating the possibility of implementing the self-management function for achieving the global control. Thus, a hierarchical and distributed architecture for the coordination and control system of the mobile elements in the robotic hand is devised. The main advantage this architecture is the very short reaction time to the control commands the device will receive from the operator, thus ensuring real-time functioning of the mobile elements in the robotic hand.

\section{The Robotic Hand Implementation}

\subsection{Functional Scheme of the Robotic System}

The components of the implemented robotic system are:

- The mechanical structure of the robotic hand having 10 degrees of freedom, with fully articulated fingers;

- The drive system of the robotic hand with adequate electric drives, controlled for gross and fine movement requirements;

- The remote control system of the robotic hand that enables transmitting in real time all the moves of the operator hand, regardless of their complexity, sensed with the multisensory control glove as sensing interface;

- The intelligent sensory interface and sensorial feedback system - contains sensory structures enabling auto-adaptive adjustment of the remote mobile elements position (as well as of the force they exert on various manipulated objects, hence generating precise and refined motion), and will allow sensory information transmission to the operators arm via a matrix of haptic (tactile) transducers. The robotic system also provides a stereoscopic video feedback by means of a stereoscopic 3D camera. Thus, the operator receives in real time the sensory feedback from the manipulation site by means of haptic transducers and specialized 3D glasses with HD OLED displays.

- Functional configurations of various complexity degrees for the robotic hand, all having complete upgrading facilities, both for the hardware and software involved; the architecture of such configurations contain different mechanical modules, as well as motor and sensory functions, according to the actual operators' requirements, being designed for subsequent-to-implement improvement and updating.

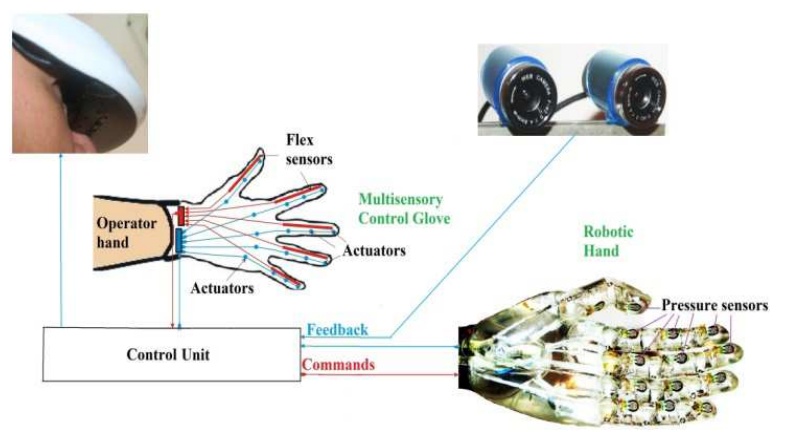

Figure 1. Schematic representation of remote controlled robotic hand with tactile feedback and control glove

The operator is equipped with the Multi-sensory control glove (MSG) and the 3D OLED HD glasses.

The MSG provides digital information on the bending degree of every finger and also on the $\mathrm{X}, \mathrm{Y}, \mathrm{Z}$ axes accelerations to the control unit. The movement control signals are serial transmitted by means of a Bluetooth module to the robotic hand, which executes the movements. Thus, the operators hand and every fingers detailed movement and position is transmitted and duplicated in real-time by the robotic hand, that executes complex human-like maneuvers controlled by the operator's hand.

The robotic hand movements are controlled by a separate microcontroller that receives the movement signals, processes them and controls the electric drives that actuate every finger, through PWM MOSFET drives. The same microcontroller also receives the pressure information from the haptic transducers placed on the robotic hands phalanxes, filters and analyses them, taking local decisions if necessary, and transmits the information wireless to the control unit. 
On every phalanx of the MSG a haptic actuator is mounted, that transmits to the operator the degree of pressure exerted on the robotic hand, by means of vibrations with varying frequency.

A stereoscopic camera is mounted on the robotic arm, transmitting continuously $3 \mathrm{D}$ visual information to the operator.

The operator perceives 3D visual information using a specialized 3D viewer (two HD OLED panels with stereoscopic glasses). In this particular experimental implementation, we have used Sony Personal 3D Viewer HMZ-T2.

\subsection{The 3D Vision Feedback}

The 3D visual monitoring is done by a remote HD stereoscopic camera (Fig. 2.b), placed in a perspective that allows intuitive control of the robotic hand. The $3 \mathrm{D}$ image is perceived by the operator by means of $3 \mathrm{D}$ viewer (Fig. 2.a,c).

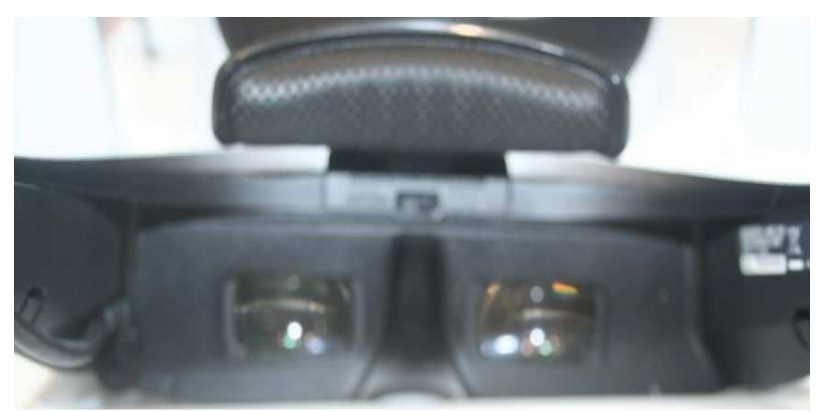

(a)

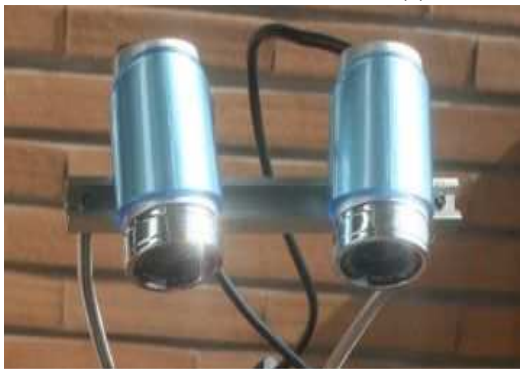

(b)

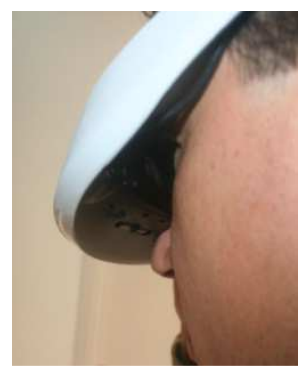

(c)

Figure 2. The $3 D$ vision feedback: $3 D$ viewer's lenses (a), HD binoculary camera (b), operator using $3 D$ viewer (c).

The high resolution and reduced latency of the video image, the minimal lag in the mechanical and computer processing of the movement and response, and very low optical distortion due to camera lens and head mounted display lenses result in an accurate and easy control of the robotic hand, the latency of the whole system being very low, practically unperceivable by the operator.

\subsection{Haptic Feedback}

The realized experiments include innovative ideas for providing the robotic hand with a matrix structure of pressure sensors, which are fitted on each segment of the robotic hand. A matrix of pressure sensors with increased complexity was implemented, using pressure sensors on each phalanx, thus offering complex information on the interactions with the maneuvered objects. Such data enables the dynamic setting and acting upon the vectorial characteristics of movements throughout the interaction with complex objects of different shapes. Based on this information, feedback elements are implemented in the MSG in order to allow the operator to sense the interactions of the robotic hand with the objects. Intelligent sensorial interfaces grant the robotic hand a large degree of adaptability and self-management, so as to enable real time response to complex stimuli and commands, coming from the user or from the environment where it operates. Therefore, by intelligent and adaptive integration of the matrix architecture of pressure sensors, the robotic hand is controlled by the operator precisely, efficiently and in real time.

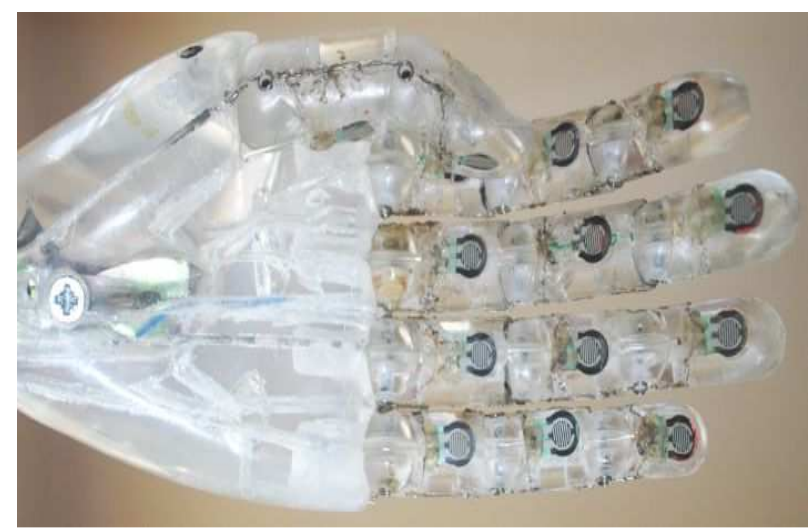

(a)

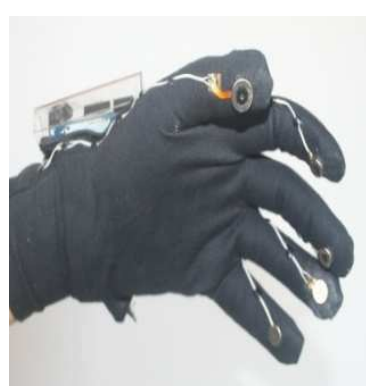

(b)

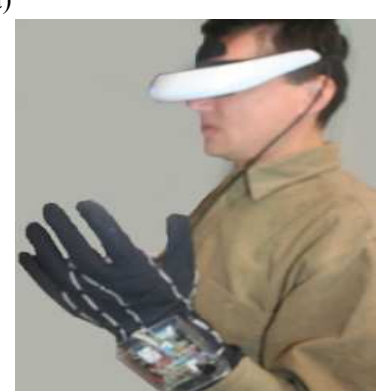

(c)
Figure 3. Haptic feedback system: the robotic hand with pressure sensors (a), MSG with haptic actuators (b), operator using the MSG and $3 D$ viewer (c).

For implementing the haptic feedback, structures of tactile actuators fitted on the interior side of the glove are used. Each tactile actuator will comes in contact with a similar area of operators hand/fingers, and generates tactile sensations with specific frequency of vibrations, depending on the pressure force exerted by the manipulated objects on the pressure sensors. In this way, information regarding the pressure force (measured by the pressure sensors) on each finger of the robotic hand will be sent to the operator hand in real time. Depending on the frequency of the vibrations applied in similar positions on the operator fingers, the operator will sense in real time the counter-force exerted by the manipulated object at the level of each finger. 


\subsection{The Mobile Platform Model}

An experimental model of a vehicle for robotic hand movement was used. It is wireless driven based on information from accelerometers included inside the MSG.

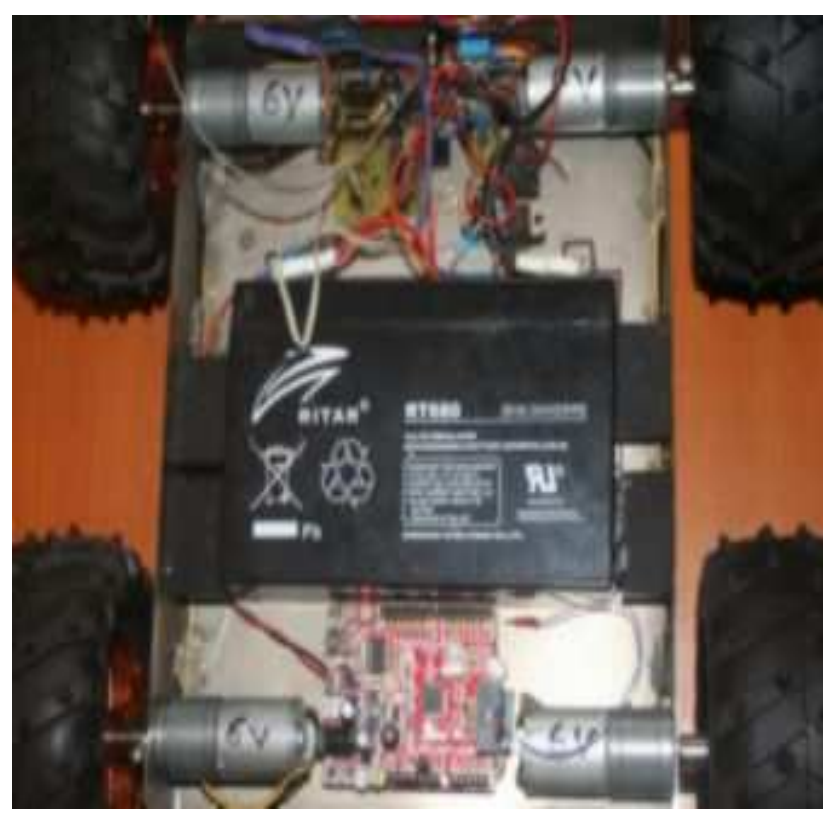

(a)

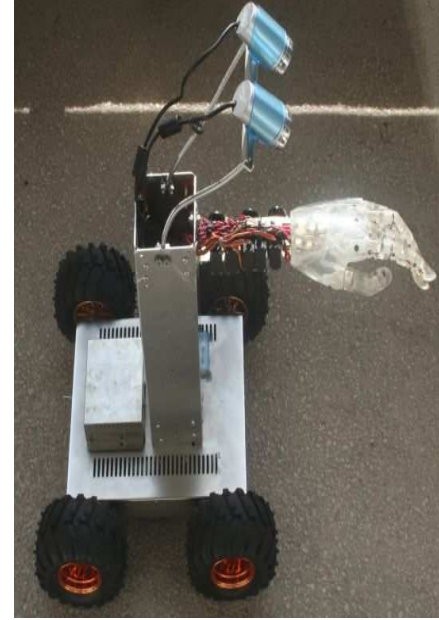

(b)

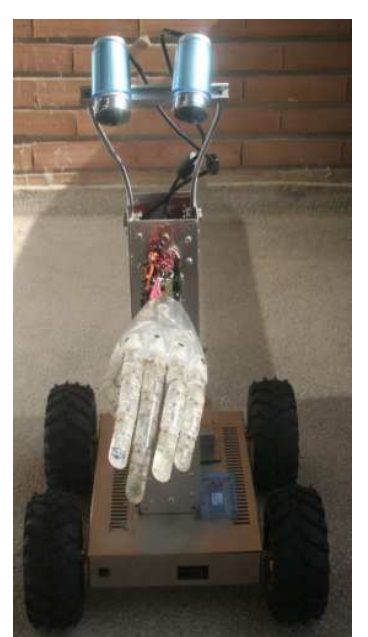

(c)
Figure 4. The mobile platform model for robotic hand transport, with robotic hand and HD cameras: the comand and power unit (a), lateral view (b), front view (c).

The robotic system can be adapted for various applications, with suitable materials and sensors. For space applications especially high radiation levels, zero gravity and zero atmospheric pressure are to be considered.

\section{Results}

The figures below show certain preliminary promising results, achieved by the partners in this project, using a simple, plastic model.

The results were obtained using an experimental robotic hand equipped with one pressure sensor on each phalanx. These experiments assisted in monitoring the pressure force on each and every sensor while objects were being maneuvered by the artificial hand.

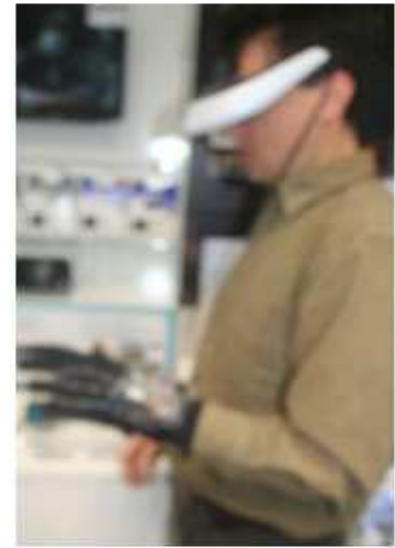

(a)

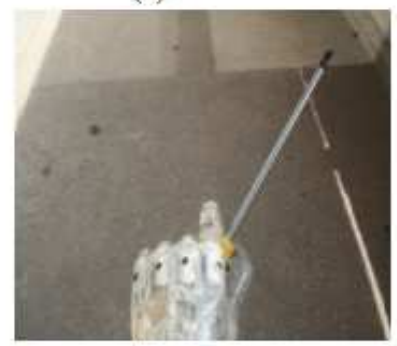

(c)

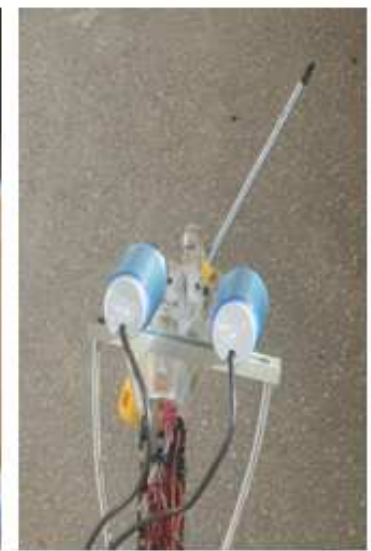

(b)

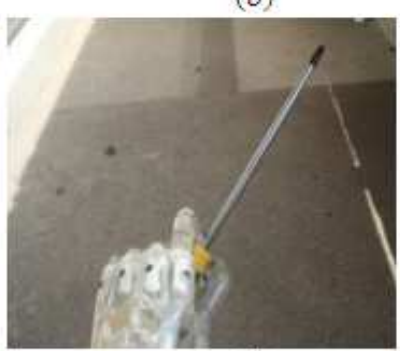

(d)
Figure 5. Experiments with the remote controled robotic hand: operator with feedback and control interfaces (a), robotic hand with a tool and binocular HD camera (b), left camera vision (c), right camera vision (d).

\section{Conclusion}

The implemented robotic system comprising a robotic anthropomorphic hand aims to achieve advanced teleoperation and telepresence functions, suitable for hazardous environment applications.

The human operator remains in a safe location and executes complex maneuvers at the dangerous site by means of the remotely controlled robotic system. The teleoperation function uses as human - machine interface a Multisensory control glove.

The telepresence function comprises haptic and 3D-vision feedback. Haptic feedback is realized through vibrations perceived by the operator directly into the glove and 3D-vision through specialized 3D glasses. The result is an easy, intuitive control of the robotic system, even for an untrained operator, with the possibility of executing high precision maneuvers.

Resolution, latency of the video image, lag in the mechanical and computer processing of the movement and response, and optical distortion due to camera lens and head mounted display lenses, have been experimentally proven as being operational, resulting in an accurate and easy control of the robotic hand. 


\section{Acknowledgements}

"This work was supported by a grant of the Romanian National Authority for Scientific Research, Programme for research - Space Technology and Advanced Research STAR, project number 82/2013."

\section{Nomenclature}

\section{References}

[1] G. Magnani, P. Rocco, L. Trevisan, A.M. Zanchettin, A. Rusconi, "Torque control in the joint of a space robotic arm", 5th IEEE International Conference on Mechatronics - ICM 2009, Malaga, Spain, April 2009.

[2] K. Landzettel, C. Preusche, A. Albu-Schäffer, D. Reintsema, B. Rebele, G. Hirzinger, Robotic On-Orbit Servicing - DLR's Experience and Perspective. In proc. of the International Conference on Intel ligent Robots and Systems (IROS), Peking, China, 2006

[3] Sommer, B. (2003). Automation and Robotics in the German Space Program - Unmanned on-Orbit Servicing (OOS) \& the TECSAS Mission. In proc. of 55th International Astronautical Congress, Vancouver, Canada, Oct. 2004

[4] Billman, D., Feary, M., \& Rochlis Zumbado, J. (2011). Evidence Report: Risk of Inadequate Design of Human and Automation/Robotic Integration. Houston, Texas: Lyndon B. Johnson Space Center

[5] Eduard Franti, Gheorghe Stefan, Paul Schiopu, Tiberiu Boros, Anca Plavitu, Intelligent control system for artificial arms configuration, Published in Proceedings of the 5th European conference on European computing conference, Pages 312-316, ISBN: 978-960-474-297-4

[6] Erhan Oztop, David W. Franklin, Thierry Chaminade, Gordon Cheng, Human-Humanoid Interaction: Is a Humanoid Robot Percieved as a Human?, International Journal of Humanoid Robotics 2(4)(2005) 537-559

[7] Christoph Bartneck, Takayuki Kanda, Omar Mubin, Abdullah Al Mahmud. (2009). Does the Design of a Robot Influence Its Animacy and Perceived Intelligence? Int J Soc Robot (2009) 1: 195-204

[8] Chintamani, K., Nawab, A., Pandya, A., Ellis, R.D., \& Cao, A. (2006). Comparing Two Kinematics Methods For Telerobotic Control Applications, Paper presented at the 50th Annual meeting of the Human Factors and Ergonomics Society, San Francisco CA.

[9] Eduard Franti, Gheorghe Stefan, Paul Schiopu, Anca Plavitu, Tiberiu Boros, Modular software for artificial arms design, Published in Proceedings of the 13th WSEAS international conference on Automatic control, modelling \& simulation, Pages 387-391, ISBN: 978-1-61804-004-6

[10] Ambrose, R., Wilcox, B., Reed, R., Matthies, L., Lavery, D., \& Korsmeyer, D. (2012). Robotics, Tele-robotics and Autonomous Systems Roadmap, Technology Area 04. Washington, DC National Aeronautics and Space Administration.

[11] Chintamani, K., Cao, A., Ellis, R.D., \& Pandya, A.K. (2010).
Improved Tele-manipulator Navigation during Display-Control Misalignments using Augmented Reality Cues. IEEE Transactions on Systems, Man and Cybernetics, Part (A), 40, 29-39.

[12] Jessie Y. C. Chen, Ellen C. Haas, and Michael J. Barnes Human Performance Issues and User Interface Design for Teleoperated Robots. IEEE Transactions on Systems, Man and Cybertenics. Part C:Applications and reviews, VOL. 37, NO. $6,11,2007$

[13] A. Birk, S. Schwertfeger, and K. Pathak, "A networking framework for teleoperation in safety, security, and rescue robotics (SSRR)," IEEE Wireless Commun. (Special Issue on Wireless Communications in Networked Robotics), vol. 6, no. 13, pp. 6-13, 2009.

[14] Curtis W. Nielsen, Michael A. Goodrich, and Robert W. Ricks, Ecological Interfaces for Improving Mobile Robot Teleoperation, IEEE Transactions on Robotics, vol. 23, no. 5, pp. 927-941, October 2007, ISSN: 1552-3098

[15] Kubota, N.; Obo, T.; Fukuda, T. "An intelligent monitoring system based on emotional model in sensor networks", Robot and Human Interactive Communication, 2009. RO-MAN 2009. The 18th IEEE International Symposium on, On page(s): 346 - 351

[16] Keshav Chintamani, R. Darin Ellis, Chin An Tan, Abhilash Pandya, Automated Augmented Reality Operator Aids for Space Robotic Teleoperations, Study funded by NASA Cooperative Agreement NNJ04HI11, 2012.

[17] K. Chintamani, A. Cao, R.D., Ellis, C.A. Tan, A.K. Pandya, Systematic Teleoperation with Augmented Reality Path Planner Navigation Cues in Cluttered Environments. Paper presented at the 53rd Annual Meeting of the Human Factors and Ergonomics Society, San Antonio, TX, 2009

[18] N. Kubota, S.Wakisaka, A. Yorita, "Tele-operation of robot partners through iPod touche", Computational Intelligence and Intelligent Informatics, 2009. ISCIII '09. 4th International Symposium on, On page(s): 75 - 80

[19] G. Ferretti, G. Magnani, P. Porrati, G. Rizzi, P.Rocco, A. Rusconi, "Real-Time Simulation of a Space Robotic Arm", Workshop on robot simulators at the IEEE/RSJ International Conference on Intelligent RObots and Systems - IROS 2008, Nice, France, September 2008.

[20] A. Rusconi, P. Magnani, A. Della Pietà, P. Campo, G. Magnani, G. Visentin, "DEXARM Integration and Test Results”, i-SAIRAS 2010, , Sapporo, Japan, pp. 354-361

[21] A. Rusconi, P. Magnani, J. F. Gonzalez Lodoso, P.Campo, R. Chomicz, G. Magnani, "Design and Development of an Integrated Joint for the Dextrous Robot Arm", 9th ESA Workshop on Advanced Space Technologies for Robotics and Automation - ASTRA 2006, ESA/ESTEC, Noordwijk (The Netherlands), November 2006.

[22] G. Hirzinger, K. Landzettel, B. Brunner, M. Fischer, C. Preusche and D. Reintsema, et al., DLR's robotics technologies for on-orbit servicing, Advanced Robotics 18(2), 139-174 (2004).

[23] K. Saitoh, T. Machida, K. Kiyokawa, and H. Takemura, A 2D-3D Integrated Interface for Mobile Robot Control Using Omnidirectional Images and 3D Geometric Models 2006. ISMAR 2006. IEEE/ACM International Symposium on, 2006. 